\title{
Research on the Visual Stimulator of Steady-State Visual-Evoked Potential Based on Android
}

\author{
Jian Gao \\ 1 Tianjin Key Laboratory of Information Sensing and Intelligent Control, Tianjin University of Technology and Education No.1310,Dagu \\ South Road, Hexi District, Tianjin 300222, China
}

\begin{abstract}
According to the brain computer interface (BCI) system requirements of steady-state visual-evoked potential, visual stimulator that based on the Android system is designed with the development platform of Android. The visual stimulator is composed of 12 stimulation modules with different frequencies. The users can represent their intentions by selecting different stimulation modules with different frequency flickers. On the basis of Android visual stimulator, EEG acquisition experiment is designed, and through the experimental verification, we come to the conclusion that visual stimulation can effectively induce the steady-state visual-evoked potential with steady and obvious features, and furthermore verify that visual stimulator has good feasibility and reliability as well as high application value.
\end{abstract}

Keywords: visual stimulator, brain-computer interface, Android.

\section{Introduction}

Brain - Computer Interface (BCI) is a new way for information exchange and control, it establishes a direct communication and control channel that does not depend on the peripheral nerve and muscle tissue between the human brain and the external environment through computer or other electronic devices. One of the most important purposes of BCI technology is to help patients who suffered from severe physical disabilities and communication disorders to achieve communication with the outside environment. Some elderly and disabled person can' $t$ communicate with the outside world with language or body due to various reasons, but BCI can provide a new way for these people to realize their needs of communicating with the outside world.

As an important part of BCI system based on steady-state visual- evoked potential, visual stimulator is also the man-machine interface in BCI system except of providing visual stimulation for the system. In accordance with this requirement, the article has designed a visual stimulator based on Android steady-state visual-evoked potential, the visual stimulator is composed of 12 stimulate modules with different frequencies. Stimulate modules with different frequency flickers represent different control commands. On the basis of Android visual stimulator, EEG acquisition experiment is designed, and then through a series of algorithms, such as preprocessing, feature extraction and pattern classification, the EEG can be converted to control command and control the external devices, thus confirming the designed visual stimulator is feasible.

\section{The Work Principle of BCI System based on Steady-State Visual-Evoked Potential}

BCI system that based on steady-state visual-evoked potential refers to a system that EEG can be treated and converted to control command, so as to realize the control on peripheral equipment, of which, EGG is generated by visual stimulation with different frequencies and different stimulus patterns; system framework is shown in Figure 1.1. Its working principle is to provide a visual stimulator that contains multiple of selectable visual stimulation targets for subjects, as visual stimuli of different frequencies represent different control commands, so the control command that subjects need to be executed can represent the stimulation target of such commands; at this time, by collecting and processing the EEG of subjects, we can determine the control commands that the subjects wanted to executed based on the induced steady-state visual-evoked potential. During the whole process, subjects, physical action and language are not required.

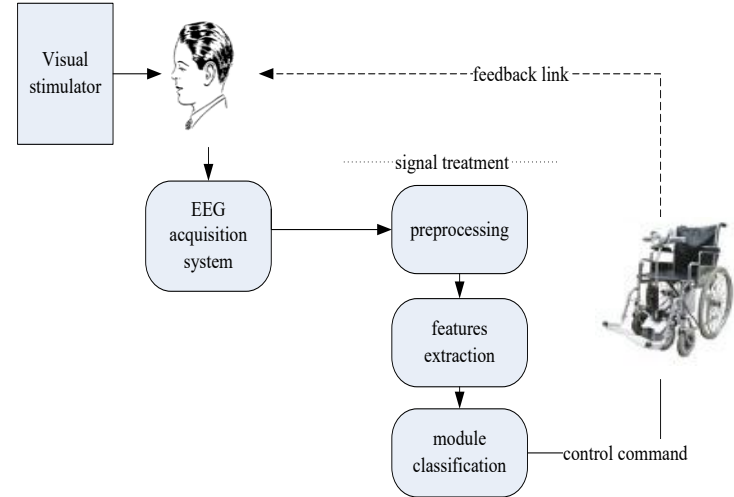

Figure 1: BCI system architecture diagram that based on steady-state visual evoked

\section{Design of Visual Stimulator}

\subsection{The Overall Design of Visual Stimulator}

Visual-evoked potential stimulator that based on Android system is composed of 12 stimulation modules of different frequencies. The flash stimulation is white lighting, and the 12 stimulation modules are arranged in the entire screen in the form of $3 \mathrm{X} 4$. Stimulation frequency is $13 \mathrm{~Hz}, 13.5 \mathrm{~Hz}, 14 \mathrm{~Hz}$, $14.5 \mathrm{~Hz}, 15 \mathrm{~Hz}, 15.5 \mathrm{~Hz}, 16 \mathrm{~Hz}, 16.5 \mathrm{~Hz}, 17 \mathrm{~Hz}, 17.5 \mathrm{~Hz}, 18 \mathrm{~Hz}$ and $18.5 \mathrm{~Hz}$. Subjects shall select the appropriate frequency of stimulation module according to the targets to be controlled. 


\section{International Journal of Science and Research (IJSR) \\ ISSN (Online): 2319-7064}

Index Copernicus Value (2013): 6.14 | Impact Factor (2014): 5.611

\subsection{Minimum Frequency Spacing}

According to the needs of the system, the designed visual stimulator contains 12 stimulation modules of different flicker frequencies; in addition, in order to make sure the visual stimulator can induce the steady-state visual-evoked potential EEG required by the experiment stably, it is necessary the frequency of each stimulation module can reach to the requirements of frequency resolution, and can distinguish the adjacent spectral peaks clearly. Therefore, we need to calculate the minimum frequency spacing of two frequencies that possessed with different EEG, the calculation is as follows:

$$
F=\frac{f_{m}}{N}
$$

$\mathrm{F}$ is the minimum frequency spacing, fm is the sampling frequency, $\mathrm{N}$ is the acquired data samples during EEG processing. The system has applied EEG amplifier that produced by Zhongke Xingtuo, with the sampling frequency of 200, so the data count that involved in each calculation of EEG is 400. According to the calculation formula for the minimum frequency spacing, we may derive frequency resolution is $0.5 \mathrm{~Hz}$. Therefore, the minimum frequency spacing of stimulation modules between two adjacent EEG stimulators is $0.5 \mathrm{~Hz}$.

\subsection{Design examples of visual stimulator}

Visual stimulator is designed based on the steady-state visual-evoked potential in Android software program. In Android application, the interface can be realized through an Activity; in the visual-evoked potential stimulator program that based on Android system, the main interface is an Activity; similarly, the flash and graphics stimulation is also an individual Activity. Moreover, two or more independent interfaces skipping can be achieved through Internet. The program flow of visual stimulator is shown in Figure 3.

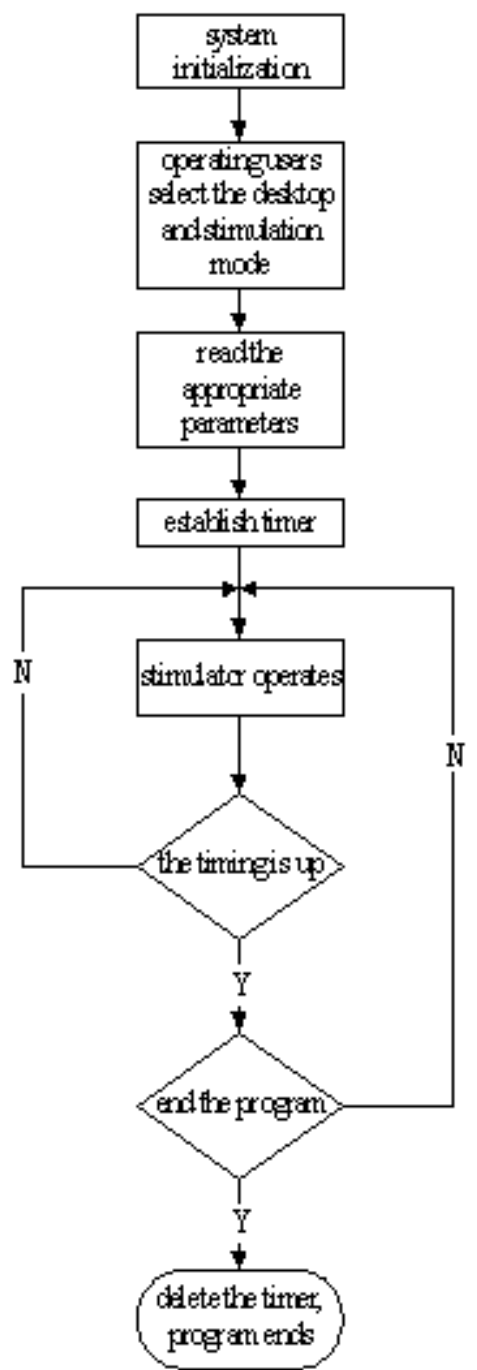

Figure 2: Program flow chart of Android visual stimulator

In order to induce the steady-state visual-evoked potential stably, a certain distance must be retained among the stimulation modules of different stimulation frequencies. Furthermore, in order to achieve more obvious of stimulus effect, subjects have to put their attentions on the stimulator, and the color of stimulation module set to be white, the color of stimulator background set to be black. Figure 4 is the physical map of multi-frequency flash stimulator.

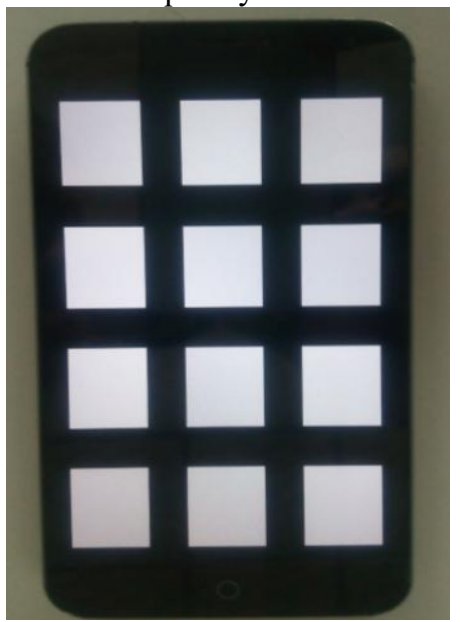

Figure 3: Physical map of multi-frequency flash stimulator 


\section{International Journal of Science and Research (IJSR) \\ ISSN (Online): 2319-7064}

Index Copernicus Value (2013): 6.14 | Impact Factor (2014): 5.611

\section{Experimental Design of Visual Stimulator}

The main purpose of research on Android-based visual stimulator is to study an emerging yet convenient visual stimulator. However, will the designed visual stimulator stably induce the EEG of steady-state visual-evoked potential, how the effect turned to be still need experimental verification. Therefore, this article has designed a confirmatory experiment of visual stimulator, and the experiments that used include: NT9200 series of EEG amplifier produced by Zhongke Xintuo, $\mathrm{Ag}-\mathrm{AgCl}$ electrode for EEG acquisition. Experimental procedure is as follows:

(1) Subjects are asked to sit on a $50 \mathrm{~cm}$ height chair right ahead of visual stimulator, stay relaxed. Distance between the eyes to the visual stimulator is about $50 \mathrm{~cm}$, and the eyes have to look at the stimulator horizontally.

(2) Place electrodes for the subjects. The acquisition position of EEG is $\mathrm{O} 1$ at head occipital region. Both earlobes are selected as a fitting position for reference electrode. Alcohol wiping is suggested before the placement, and then solid conductive paste is placed at the electrodes of occipital region, while the reference electrodes at earlobes shall be injected with conductive fluid.

(3) Turn on the computer, run the EEG data storage system program that designed with LabVIEW platform and click the EEG amplifier.

(4) Remind subjects the experiment is about to begin, please stat focus.

Experiment has collected $13 \mathrm{~Hz}, 14 \mathrm{~Hz}, 15 \mathrm{~Hz}$ and $16 \mathrm{~Hz}$ EEG with four different frequencies. EEG preprocessing and feature acquisition are completed on the computer, the power spectrum after EEG signal processing displays that the identification effect of EEG with four different frequencies is very clear, from where we can see the designed visual stimulator can induce effective steady-state visual-evoked potential EEG.
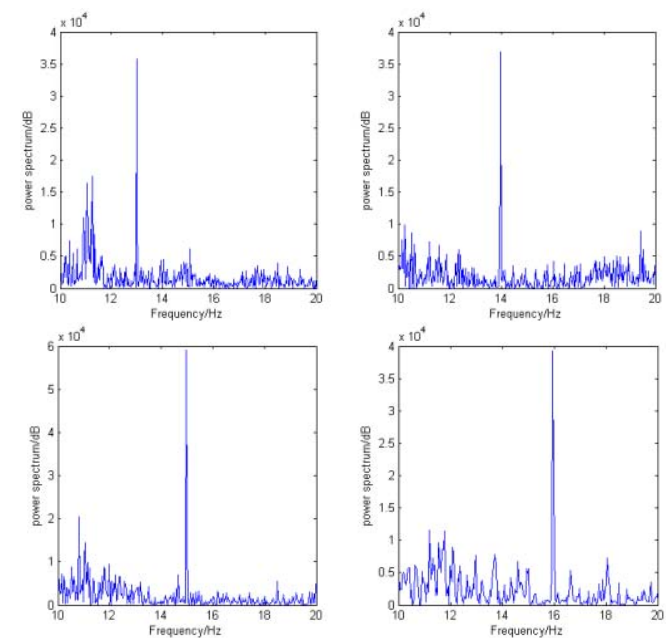

Figure 5 : $13 \mathrm{~Hz}, 14 \mathrm{~Hz}, 15 \mathrm{~Hz}, 16 \mathrm{~Hz}$ EEG power spectrum is widely used in the BCI system as an input signal. Visual stimulator is applied as peripheral equipment of visual stimulus in BCI system based on steady-state visual-evoked potential, so it is both the human-computer interaction, and the prerequisite and guarantee of recording EEG data. In this paper, the designed visual stimulator that based on steady-state visual-evoked potential of Android system can be regarded as an exploration and research, as the conventional visual stimulator is quite complex and not easy to move. Meanwhile, it is also an attempt to combine Android system and BCI two hot technologies. I believe along with the further study on BCI and additional research results, the BCI system will be widely used.

\section{References}

[1] Guan Jin'an, Zhang Tengfei, Zeng Xingtao. EEG Recorder Design based on Android mobile device [J] Academic Journal of South-Central University for Nationalities, 2014.3 Vol 33 (1): 73-76.

[2] Xie Hong, Qian Menchao. Android-based EEG wireless acquisition and analysis system design [J] Application Wonder, 2014 Vol 33 13:. 77-80.

[3] Zheng Jun. BCI realization and research based on steady-state visual-evoked potential[D] Guangzhou: South China University of Technology, 2012.

[4] Zhu Weifang. FPGA-based research on multi-function evoked potential stimulator [D] Chongqing: Chongqing University, 2012.

[5] Cheng Guanghui. Research on the achieve technology of BCI visual stimulator [D] Chongqing: Chongqing University, 2006.

[6] Xiao Xiaojun. BCI visual stimulator design [D] Beijing: China University of Geosciences, 2009.

[7] Guo Xuhong. Research on asynchronous BCI system based on multi-movement imagine[D] Tianjin: Tianjin Vocational and Technical Normal University, 2014.

[8] Zhu Weifang, Chen Ji. The development of a multifunctional visual evoked potential stimulator $[\mathrm{J}]$ Application of Electronic Technique, 2012,38 (6): 54-57

\section{Author Profile}

Jian Gao is reading the M.S. degrees in Tianjin University of Technology and Education .

\section{Experimental Design of Visual Stimulator}

EEG of steady-state visual-evoked potential is easier to extract and more stable compared to other types of EEG, so it 